\title{
Neurometabolic disorder due to serine deficiency
}

INSERM

\section{Source}

INSERM. (1999). Orphanet: an online rare disease and orphan drug data base.

Neurometabolic disorder due to serine deficiency. ORPHA:35705

Serine-deficiency syndrome is a very rare infantile-onset potentially treatable neurometabolic disorder characterized clinically by microcephaly, neurodevelopmental disorders and seizures. Three serine-deficiency syndromes have been described: 3phosphoglycerate dehydrogenase (3-PGDH) deficiency, 3-phosphoserine phosphatase (3-PSP) deficiency, and phosphoserine aminotransferase deficiency (see these terms). 